\title{
Baculovirus immediately early 1 , a mediator for homologous regions enhancer function in trans
}

Xu'ai Lin ${ }^{1,2}$, Yin Chen ${ }^{2}$, Yongzhu Yi ${ }^{2}$, Zhifang Zhang ${ }^{2 *}$

\begin{abstract}
Background: Enhancers are DNA sequences that serve as binding sites for regulatory proteins, and stimulate transcriptional activity independent of their positions and orientations with respect to the transcriptional initiation site. Previous studies considered that baculovirus homologous regions (hrs) function as enhancers in cis. In our study, a plasmid containing homologous region 3 (hr3) enhancer from Bombyx mori nucleopolyhedrovirus (BmNPV) failed to enhance transcription of promoter in other plasmid in co-transfection assays, but strong stimulation occurred when cells were infected by BmNPV.

Results: The cotransfection results of each BmNPV genomic library plasmid, hr3 plasmid and reporter plasmid showed that there were eight library plasmids stimulated the luciferase gene expression remarkably. Sequencing these plasmids revealed that each of them contained the ie-1 gene. Transfected plasmids, containing ie-1, hr3 and various origin promoter drove reporter gene showed the function was even retained. Cotransfection of hr3 functional dissected fragment and ie-1 revealed that the 30-bp imperfect palindrome destroyed fragment can't enhance reporter gene expression even though transfected with ie-1.

Conclusion: IE-1 was the only early factor of BmNPV that could act as a mediator for hr enhancer function in trans and the trans-function was achieved with a broad-spectrum of promoters. The 30-bp imperfect palindrome was the elementary molecular structure by which IE-1 participated in the enhancer function in trans.
\end{abstract}

\section{Background}

The genome of baculovirus contains interspersed homologous regions ( $h r s)$ that function as transcriptional enhancers linking in cis to viral or heterologous promoters in either insect or mammalian cells [1]. The immediately early gene $1, i e-1$, is one of six essential genes required for DNA replication in transient replication assays, and the $67-\mathrm{kDa}$ encoded product of $i e-1$ is the principal transcriptional regulator of baculovirus [2]. As assayed by plasmid transfection, IE- 1 transactivates the expression of various baculovirus early genes and some housekeeping genes [3]. When the affected promoter links in cis to the $h r$ enhancer, IE-1 protein also markedly stimulates promoter activity through binding to the 28-mer palindrome units [4-6].

Transcriptional enhancers for eukaryotic genes are binding sites for regulatory proteins; they lie at a distance upstream or downstream of the transcriptional

\footnotetext{
* Correspondence: zhangzf@caas.net.cn

${ }^{2}$ Biotechnology Research Institute, Chinese Academy of Agricultural Sciences, Beijing 100081, China
}

start sites, and the regulatory proteins that bind to them activate (or sometimes inhibit) transcription $[7,8]$. A previous report showed that the $h r$ enhancer stimulated transcription only in the cis-linked conformation [9]. In contrast, another study found that when plasmid p39CAT was co-transfected with BglII-digested viral DNA and a PstI DNA library of Autographa californica Nucleopolyhedrovirus (AcNPV), the CAT activity increased remarkably [10].

In our study, $h r 3$ from BmNPV failed to enhance the expression of the luciferase gene (luc) in trans in cotransfection assays, but strong enhancement occurred when the two independent plasmids were co-transfected into silkworm cells along with BmNPV. Therefore, we assumed that certain viral factor(s) participate in the trans-activation effect. A random BmNPV genomic library was constructed and used to screen viral factor (s) mediating $h r 3$ enhancer function in trans through co-transfection with DNAs from reporter plasmid and $h r 3$ enhancer-containing plasmid. According to the structural characteristics of the $h r 3$ enhancer, dissection 
analyses with different amounts of palindromes were conducted to uncover the basic requirement for $h r 3$ enhancer function in trans.

\section{Methods \\ Materials}

T4 DNA ligase, platinum $p f x$ DNA polymerase and the lipofectin kit were purchased from Invitrogen (USA). Taq DNA polymerase, restriction endonucleases, pGEM-T easy vector, DNA purification kit, luciferase assay kit and pRL-CMV vector for internal control transfections were purchased from Promega Corp (USA). E. coli strain DH10B was maintained in our lab. The reporter plasmids pKS-hel510-luc, pKS-Bmgp64-luc and pGEM3Z-lsp-luc, containing helicase, gp 64 and the silkworm larvae serum protein $(l s p)$ gene promoter respectively, were from our previous work [11-13]. The enhancer vectors, pKS-hr114, pKS-hr198 and pKS-hr3 containing 0,1 or 3 30-bp incomplete palindromes respectively, were constructed and maintained in our lab [14].

\section{Virus, cell lines and random library}

The BmNPV-ZJ8 strain was maintained in our lab. $\mathrm{Bm}-\mathrm{N}$ cells were propagated at $27^{\circ} \mathrm{C}$ in TC-100 insect medium supplemented with $10 \%$ heat-inactivated $\left(56^{\circ} \mathrm{C}\right.$, $30 \mathrm{~min}$ ) fetal bovine serum (FBS) (Invitrogen). The details for cell culture were from Summers and Smith's manual [15]. A random genomic library of BmNPV was constructed according to the "partial filling-in" method that contained a $3 \mathrm{~kb}$ to $5 \mathrm{~kb}$ fragment in the pUC19 vector [16,17]. Plasmid DNAs of 238 positive colonies were extracted for further transient assays [17].

\section{Transfection in insect cells}

$\mathrm{Bm}$-N cells were seeded in 24-well plates and allowed to attach at $27^{\circ} \mathrm{C}$ overnight. Transfection assays were conducted using lipofectin following the manufacturer's instructions. The co-transfection solution contained 0.3 $\mu \mathrm{g}$ reporter plasmid DNA, $0.1 \mu \mathrm{g}$ internal control plasmid DNA in some cases, $0.3 \mu \mathrm{g}$ of each plasmid DNA from the random library, and $h r$ enhancer when necessary, along with $2 \mu \mathrm{l}$ lipofectin in a total volume of 50 $\mu \mathrm{l}$. pBlueScript DNA was introduced in some reactions to maintain a constant quantity of DNA. If virus infection was required, the virus was added to the serum-free medium and left for $1 \mathrm{~h}$ before the supernatant was replaced with complete medium. Each transfection contained at least three separate experiments.

\section{Luciferase activity assay}

The cells were harvested at $48 \mathrm{~h}$ post transfection (hpt) and cell extracts were prepared following the instructions with the luciferase assay kit (Promega). The amount of protein in the lysate was measured using the Bradford method [18]. Measurements of dual-luciferase activity were performed with a liquid scintillation spectrometer (Beckman LS6000 Series, USA) [19]. Luciferase activity was indicated as counts per minute (CPM) in 15 s.

\section{Cloning of Orf121, Orf122 and ie-1 genes}

Using BmNPV ZJ-8 DNA as template, the intact ORFs and corresponding $5^{\prime}$ untranslated region (5'-UTR) were amplified. Primers (Table 1) were designed according to the sequence of BmNPV T3 strain (GenBank accession no. L33180). The amplified fragments were subsequently cloned into the pGEM-T easy vector, and were confirmed by direct sequencing.

\section{Results}

Function of the hr enhancer in trans via virus infection

The helicase promoter of BmNPV was rather weak in transient assays, and only just-detectable luciferase activity was found. When the transfected cells were infected with BmNPV, or co-transfected with reporter plasmid and pKS-hr3, transcription of helicase promoter was slightly augmented, that is to say, $h r 3$ did not appear to function as an enhancer when presented in separate plasmids in insect cells. However, if the co-transfected cells were infected by BmNPV, luciferase activity was markedly increased, by 58447.7 -fold. This result suggested that the $h r$ enhancer stimulated the individual promoter when viral factor(s) were present even when they were presented in separate plasmids. A similar result (28454.5-fold) was obtained using the $l s p$ promoter, a eukaryotic promoter from the silkworm larva (Table 2). We assumed that certain viral factor(s), which served as mediator(s) for the hr enhancer, functioned in trans to greatly stimulate transcription.

Genome-wide screening for viral factors mediating the $\mathrm{hr}$ enhancer function in trans

Since the $h r$ enhancer functioned in trans in the presence of viral factors, a random genomic library was constructed for high-throughput, genome-wide screening of viral factors. The sreening was carried out by co-

Table 1 Primers used to amplify the orf121, orf122 and ie-1 genes, as well as 5' UTR

\begin{tabular}{ccc}
\hline Genes & Primer name & Sequences (5' $^{\prime} \mathbf{3}^{\prime}$ ) \\
\hline orf121 & Orf121-F & CACGACGCGCAGCGATGATTAC \\
& Orf121-R & GAAAGCACTCTTCATAATAACAAG \\
orf122 & Orf122-F & CAGTGGTCTTGGCAAACATTCC \\
& Orf122-R & CTTGTTATATGAAGAGTGGCTTC \\
ie-1 & le1-F & GCACAGACAAAATGTGCCACACTTG \\
& le1-R & CCAACTCCCATTGTT ATTATGCAAC \\
\hline
\end{tabular}


Table 2 Transactivation effects of $h r 3$ enhancer on target promoters via BmNPV infection in Bm-N cells

\begin{tabular}{ccccc}
\hline Reporter plasmids & pSK-hr3 & BmNPV & CPM & Fold-stimulation \\
\hline pKS-hel510-luc & - & - & $22.3 \pm 4.5$ & 1.0 \\
& + & - & $17 \pm 3.8$ & 0.8 \\
pGEM3Z-Isp-luc & - & + & $80 \pm 13.3$ & 3.6 \\
& + & + & $1303384 \pm 74692$ & 58447.7 \\
& - & - & $11.6 \pm 4.1$ & 1.0 \\
& - & + & $68 \pm 21.2$ & 1.5 \\
& - & + & $227636 \pm 37514$ & 8.5 \\
\end{tabular}

Luciferase activity indicated as CPM in $15 \mathrm{sec}$ (minus background counts of cells). Transactivation of hr3 enhancer with or without virus infection presented as stimulating fold over each corresponding reporter plasmid transfection alone, that was arbitrarily set as 1.0 . Each reaction contained $10 \mu \mathrm{g}$ of protein extracted from the transfected cells.

transfection of reporter plasmid and $h r$ enhancer, along with each member of the library. Eight plasmids, which greatly increased luciferase activity in transient expression, were screened from the random library. In contrast, without the $h r$ enhancer, each of the eight library plasmid DNA products still slightly stimulated the transcription of helicase promoter. This result suggested that each of these library plasmid DNAs contained a transactivator-coding region. Other 230-library plasmid DNAs did not stimulate helicase transcription whether $h r$ enhancer was present or not. After sequencing, the corresponding sequences inserted in the eight plasmids were aligned with the BmNPV T3 strain genome sequence, and the intact ORFs in each plasmid are listed in Table 3.

\section{IE-1 protein affected hr enhancer function in trans alone} According to the screened regions, odv-e18, odv-ec27, $o d v$-e56 and orf-125 were included in some of the eight plasmids, so we considered that these genes were not concerned with the enhancement of promoter activity, while another three genes, orf-121, orf-122 and ie-1, were all included in each of the 8 plasmids. Based on the complete genomic sequence of the BmNPV T3 strain, ORF-121 and ORF-122 are hypothetical proteins encoded by an intergenic region between IE-0 and IE-1, with molecular weights of 11 and $23 \mathrm{kDa}$ respectively. To investigate whether ORF-121, ORF-122 or IE-1 protein alone is sufficient to recover the ability of $h r$ enhancer to function in trans, orf-121, orf-122 and ie-1 with their promoter regions were cloned into pGEM-T easy vector, respectively. The recombinant plasmid was used for co-transfection assays to identify whether these three gene products participated in $h r$ enhancer function in trans. Two BmNPV-derived promoters, helicase and gp64, host-derived $l s p$ promoter and mammalian virusderived $C M V$ promoter/enhancer regions were used for the test. All the results revealed a stimulatory effect ranged from 40 to more than 100 folds as shown in table 4 when co-transfected reporter plasmid, hr3 plasmid and ie-1 plasmid. It demonstrated that the trans-function of enhancer on a broad-spectrum of promoters was achieved through the involvement of IE-1 protein while not through orf121 or orf122 protein.

\section{Functional dissection of hr enhancer structure essential for function in trans}

The 651-bp hr3 enhancer of BmNPV contains three direct repetitive regions, each of which contains a 30-bp incomplete palindrome with a naturally occurring EcoRI site as the core. The plasmid pKS-hr114 contains a 114 bp enhancer fragment from $h r 3$ with no intact 30-bp

Table 3 Plasmids involved in hr3 enhancer function in trans

\begin{tabular}{clll}
\hline Plasmid No. & Corresponding site in T3 strain & Intact coding regions contained & CPM \\
\hline 19 & $114443-119152$ & odv-e18, odv-ec27, orf-121, orf-122, ie-1 & 202023 \\
58 & $115898-119619$ & orf-121, orf-122, ie-1 & 554392 \\
186 & $115898-119152$ & orf-121, orf-122, ie-1 & 100268 \\
262 & $115216-119619$ & orf-121, orf-122, ie-1 & 112732 \\
280 & $114846-120318$ & odv-ec27, orf-121, orf-122, ie-1, odv-e56, orf-125 & 381711 \\
289 & $115216-119619$ & orf-121, orf-122, ie-1 & 261497 \\
310 & $114846-120549$ & odv-ec27, orf-121, orf-122, ie-1, odv-e56, orf-125 & 159852 \\
347 & $115898-119152$ & orf-121, orf-122, ie-1 & 717312 \\
\hline
\end{tabular}

All the coding regions were presumed according to the complete genome sequence of the BmNPV T3 strain (GenBank accession no. L33180). Luciferase activity indicated as CPM in $15 \mathrm{sec}$ (minus the background counts of cells). Each reaction contained $10 \mu \mathrm{g}$ protein extracted from the transfected cells. The CPM values obtained from the transfection of helicase promoter containing reporter plasmid alone were in the range of 18 to 40 . 
Table 4 Transactivation effects of $h r 3$ enhancer on target promoters via IE-1 protein bridge.

\begin{tabular}{ccccc}
\hline $\begin{array}{c}\text { Reporter } \\
\text { plasmids }\end{array}$ & $\begin{array}{c}\text { pGEM-T- } \\
\text { ie1 }\end{array}$ & $\begin{array}{c}\text { pSK- } \\
\text { hr3 }\end{array}$ & CPM & $\begin{array}{c}\text { Fold- } \\
\text { stimulation }\end{array}$ \\
\hline pKS-hel510-luc & - & - & $21.4 \pm 5.6$ & 1.0 \\
& + & - & $16093 \pm 1432$ & 752 \\
& + & + & $735880.1 \pm$ & 34387 \\
pSK-Bmgp64-luc & - & - & $74.9 \pm 15.4$ & 1.0 \\
& + & - & $40080 \pm 2947$ & 535 \\
pGEM3Z-Isp-luc & + & + & $5738241 \pm$ & 76612 \\
& + & - & 609222 & 1.0 \\
& + & & $208 \pm 17.4 \pm 3.9$ & 14.4 \\
pRL-CMV & + & - & $78 \pm 11.8$ & 1866 \\
& + & - & $404 \pm 53.5$ & 5.2 \\
& + & + & $76400 \pm 9312$ & 979.5
\end{tabular}

Luciferase activity indicated as CPM in $15 \mathrm{sec}$ (minus the background counts of cells). Transactivation of $\mathrm{hr} 3$ enhancer with or without ie-1 gene $(0.1 \mu \mathrm{g}$ DNA in each transfection) presented as stimulating fold over each corresponding reporter plasmid transfection alone, which was arbitrarily set as 1.0. Each reaction contained $10 \mu \mathrm{g}$ of protein extracted from the transfected cells.

incomplete palindrome but with half of the palindrome on both sides. pKS-hr198 contains one 30-bp incomplete palindrome, and pSK-hr3 contains an intact 651bp $h r 3$ fragment with 3 palindromes. The incomplete palindrome is the vital structure for $h r$ enhancer function in cis. To evaluate the effect of the palindrome on enhancer function in trans, and further understand how ie-1 participates in the enhancer function in trans, reporter plasmids were co-transfected with the $h r$ derivates, by virus infection or cotransfected with the $h r$ derivates and pGEM-T-ie1. Results revealed that the palindrome was essential to $h r$ enhancer function in trans. pKS-hr114 did not stimulate transcription from affected promoters even by virus infection or co-transfection with pGEM-T-ie1. The constructs with intact palindrome(s) dramatically increased the transcription of the reporter gene in the presence of IE-1 protein (Fig. 1 and 2).

\section{Discussion}

Baculovirus homologous regions are repeated sequences that are interspersed in the genomes of baculoviruses. It is known that these regions contain the origins of DNA replication, and augment the expression of a number of genes in an orientation-independent manner [9].

In the random genomic library of BmNPV, the average size of fragments were 3 to $5 \mathrm{~kb}$, while the largest open reading frame of BmNPV, helicase, was $3669 \mathrm{bp}$, so we ensured that the largest coding region in the genome was included in the random library. According to the average size of cloned fragments, the representation of the random library was more than 99\% [20]. Therefore, the completeness and representation of the constructed library was adequate for genome-wide screening for regulatory products or elements.

In some cases, enhancers can function in trans on a separate DNA molecule via a protein bridge to the promoter by covalent linkage of molecules or a cellular factor binding with the two elements $[21,22]$. The simian virus 40 (SV40) enhancer functions in trans to the $\beta$ globin promoter when they are linked by a protein bridge [21]. A recent report even suggests that certain enhancers can interact with several target promoters in trans on different chromosomes [23]. Genome-wide screening revealed eight plasmids, all containing orf-121, orf- 122 and ie-1 coding regions could stimulate the expression of luciferase. Subsequent experiments showed that IE-1 was the only factor of BmNPV that acted as a mediator for the $h r$ enhancer functioning in trans. This may be because the sizes of the two hypothetical proteins ORF121 and ORF122 are too small to afford the binding domain and activating domain required for a transcription factor. Previous reports using gel shift assays and mutational analyses confirm that IE-1 binds directly to the $h r$ enhancers $[24,25]$. The direct interaction between IE-1 and $h r$ enhancer may be consistent with the theory that some eukaryotic enhancers reach their targets (as yet undefined) by means of DNA loops, IE-1 bring the $h r$ enhancer to the proximal promoter and the complex is easily recruited by the transcriptional apparatus $[26,27]$. In this study, we assumed that IE-1 plays two distinct roles, one as a transactivator to stimulate the transcription of the basal promoter, and the other as a mediator for $h r$ enhancer functioning in trans by binding to the $\mathrm{hr}$ enhancer and then reaching the target promoter to give another 40 to 100 -fold activation. So the enormous enhancement of luciferase activity was obtained by the cumulative effects of these mechanisms. When hr114 was co-transfected with pGEM-T-ie1 and reporter plasmids, the CPM values were lower than control assays with co-transfected pGEM-T-ie1 and reporter plasmids. Since IE-1 can bind with a half palindrome of $h r$ enhancer, the competitive binding of IE-1 by the two half palindromes might have resulted in the decrease of IE-1 transactivator. It was reported that the imperfect palindrome, especially the naturally occurring EcoRI site, is essential for its enhancing function in cis [25]. The present study demonstrated that the intact palindrome is also the elementary structural requirement for $h r$ enhancer functioning in trans.

AcMNPV hr5 functions in trans in an IE-1-dependent $39 \mathrm{~K}$ promoter and the $p 35$ promoter, and the stimulating effects of hr5 in trans are about 2 and 7 -fold 


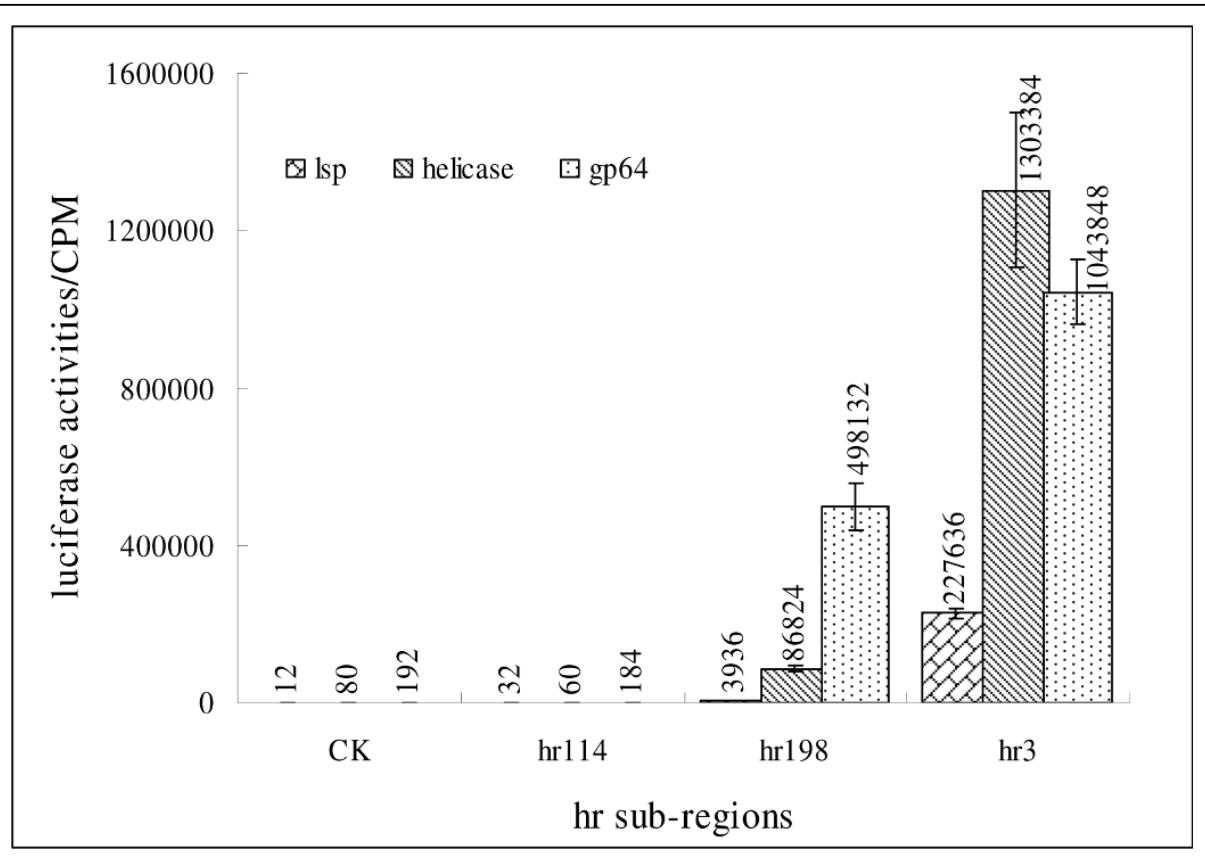

Figure 1 Transactivation effects of $h r 3$ enhancer sub-regions on different promoters via virus infection. Reporter plasmids containing different promoters were co-transfected with the $h r$ derivates by virus infection. $10 \mu \mathrm{g}$ of protein was extracted from the transfected cells to determine the luciferase activity. hr114 is a plasmid containing a fragment without an intact 30-bp incomplete palindrome but half of the palindrome on both sides; hr198 contains one 30-bp incomplete palindrome; hr3 contains an intact 651-bp hr3 fragment with 3 palindromes. CK had no hr added.

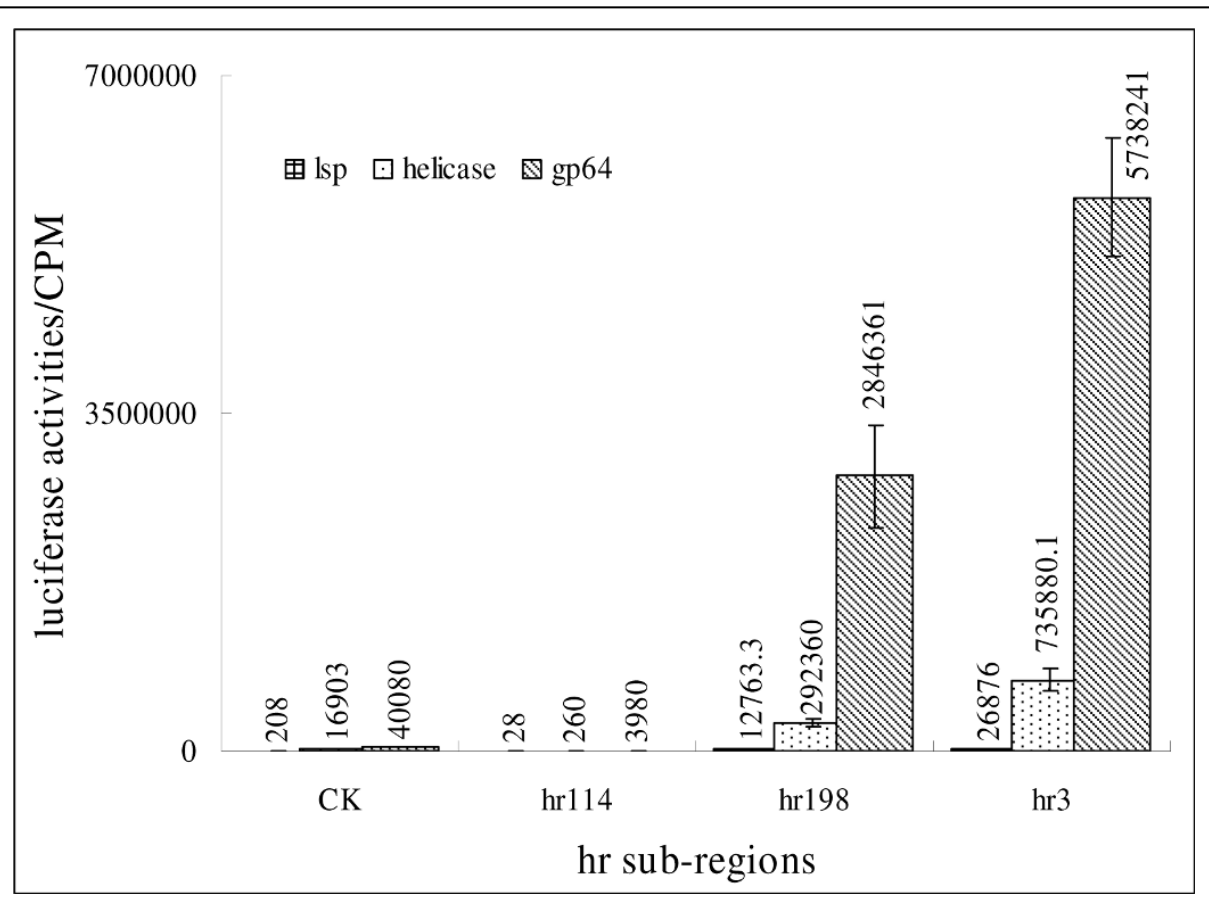

Figure 2 Transactivation effects of $h r 3$ enhancer sub-regions on different promoters via co-transfection with pGEM-T-ie1 DNA. Reporter plasmids containing different promoter were co-transfected with pGEM-T-ie1 and hr derivates. $0.1 \mu \mathrm{g}$ of pGEM-T-ie1 plasmid DNA was used in each transfection. Each luciferase reaction contained $10 \mu \mathrm{g}$ of protein extracted from the transfected cells. CK, no hr added; hr114, plasmid containing a fragment without intact 30-bp incomplete palindrome but half of the palindrome on both sides; hr198 contained one 30-bp incomplete palindrome; hr3 contained an intact 651-bp hr3 fragment with 3 palindromes. 
$[28,29]$. In this report, the IE-1 induced much higher activation of all the differently derived promoters to the trans-presented $h r 3$ enhancer from BmNPV, and this suggests that IE-1 is a generic mediator for $h r$ enhancer functioning in trans. Screening of the genomic library confirmed that IE- 1 is the only viral factor that mediates $h r$ enhancer functioning in trans. Furthermore, this result implies that in some transfection experiments, particularly for co-transfection molecules and internal controls, the trans-effects should not be ignored [30].

\section{Acknowledgements}

We thank lain C. Bruce and Gen Wang for critical reading of the manuscript. This work was supported by grants from the National Natural Sciences Foundation of China (30670082) and the "863" Project (2006AA10A119).

\section{Author details}

'Department of Medical Microbiology and Parasitology, School of Medicine, Zhejiang University, Hangzhou 310058, China. ${ }^{2}$ Biotechnology Research Institute, Chinese Academy of Agricultural Sciences, Beijing 100081, China.

\section{Authors' contributions}

$X A L$ and $Y C$ performed the experimental work and analyzed the data. YZY contributed to the cell culture. XAL, YC and ZZF conceived the experimental strategies and designed the experiments. All authors read and approved the final manuscript.

\section{Competing interests}

The authors declare that they have no competing interests.

Received: 25 November 2009

Accepted: 10 February 2010 Published: 10 February 2010

\section{References}

1. Viswanathan P, Venkaiah B, Kumar MS, Rasheedi S, Vrati S, Bashyam MD, Hasnain SE: The homologous region sequence (hr1) of Autographa californica multinucleocapsid polyhedrosis virus can enhance transcription from non-baculoviral promoters in mammalian cells. J Biol Chem 2003, 278:52564-52571.

2. Kool M, Ahrens $\mathrm{CH}$, Goldbach RW, Rohrmann GF, Vlak M: Identification of genes involved in DNA replication of the Autographa californica baculovirus. Proc Natl Acad Sci USA 1994, 91:11212-11216.

3. Lu M, Johnson RR, latrou K: Trans-activation of a cell housekeeping gene promoter by the IE1 gene product of baculoviruses. Virology 1996, 218:103-113.

4. Lu A, Carstens EB: Immediate-early baculovirus genes transactivate the p143 gene promoter of Autographa californica nuclear polyhedrosis virus. Virology 1993, 195:710-718.

5. Pullen SS, Friesen PD: Early transcription of the IE-1 transregulator gene of Autographa californica nuclear polyhedrosis virus is regulated by DNA sequences within its 59 noncoding leader region. J Virol 1995, 69:156-165.

6. Rodems SM, Pullen SS, Friesen PD: DNA-dependent transregulation by IE1 of Autographa californica nuclear polyhedrosis virus: IE1 domains required for transactivation and DNA binding. J Virol 1997, 71:9270-9277.

7. Guarente L: UASs and enhancers: common mechanism of transcriptional activation in yeast and mammals. Cell 1988, 52:303-305.

8. Maniatis T, Goodbourn S, Fischer JA: Regulation of inducible and tissuespecific gene expression. Science 1987, 236:1237-1245.

9. Lu M, Farrell PJ, Johnson R, latrou K: A baculovirus (Bombyx mori nuclear polyhedrosis virus) repeat element functions as a powerful constitutive enhancer in transfected insect cells. J Biol Chem 1997, 272:30724-30728.

10. Carson DD, Guarino LA, Summers MD: Functional mapping of an AcNPV immediately early gene which augments expression of the IE-1 transactivated 39K gene. Virology 1988, 162:444-451.

11. Xiao QL, Zhang ZF, Yi YZ, He JL, Wu XF: Functional analysis of helicase gene promoter and homologous region 3 enhancer in Bombyx mori nuclear polyhedrosis virus. Acta Biochim Biophys Sin 2001, 33:525-530.
12. Zhou YJ, Yi YZ, Zhang ZF, He JL, Zhang YX, Wu XF: Promoter activities in the baculovirus envelope glycoprotein gp64 gene. Acta 2003, 35:18-26.

13. Tang SM, Yi YZ, Shen XJ, Zhang ZF, Li YR, He JL: Functional analysis of the larval serum protein gene promoter from silkworm, Bombyx mori. Chinese Sci Bull 2003, 48:2611-2615.

14. Chen Y, Yao B, Zhu ZZ, Yi YZ, Lin XA, Zhang ZF, Shen GF: A constitutive super-enhancer: homologous region 3 of Bombyx mori nucleopolyhedrovirus. Biochem Biophys Res Commun 2004, 318:1039-1044.

15. Summers MD, Smith GE: A manual of methods for baculovirus vectors and insect cell culture procedures. Texas Agric Exp Stat Bull No.1555 1987.

16. Zabarovsky ER, Allikmets RL: An improved technique for the efficient construction of gene libraries by partial filling-in of cohesive ends. Gene 1986, 42:119-123.

17. Chen Y, Lin XA, Yi YZ, Lu YY, Zhang ZF: Construction and application of a baculovirus genomic library. Z Naturforsch [C] 2009, 64c:574-580.

18. Smith JA: Analysis of proteins. Short Protocols in Molecular Biology New York: John Wiley \& Sons IncAusubel FM, Brent R, Kingston RE, Moore DD, Seidman JG, Smith JA, Struhl K, 3 1995, 332-333.

19. Idahl LA, Sandstrom PE, Sehlin J: Measurements of serum glucose using the luciferin/luciferase system and a liquid scintillation spectrometer. Anal Biochem 1986, 155:177-181.

20. Seed B, Parker RC, Davidson N: Representation of DNA sequences in recombinant DNA libraries prepared by restriction enzyme partial digestion. Gene 1982, 19:201-209.

21. Müeller-Storm HP, Sogo JM, Schaffner W: An enhancer stimulates transcription in trans when attached to the promoter via a protein bridge. Cell 1989, 58:767-777.

22. Mahmoudi T, Katsani KR, Verrijzer CP: GAGA can mediate enhancer function in trans by linking two separate DNA molecules. EMBO J 2002, 21:1775-1781.

23. Lomvardas S, Barnea G, Pisapia DJ, Mendelsohn M, Kirkland J, Axel R: Interchromosomal interactions and olfactory receptor choice. Cell 2006, 126:403-413.

24. Choi J, Guarino LA: The baculovirus transactivator IE1 binds to viral enhancer elements in the absence of insect cell factors. J Virol 1995, 69:4548-4551.

25. Rodems SM, Friesen PD: Transcriptional enhancer activity of hr5 requires dual-palindrome half sites that mediate binding of a dimeric form of the baculovirus transregulator IE1. J Virol 1995, 69:5368-5375.

26. Popham DL, Szeto D, Keener J, Kustu S: Function of a bacterial activator protein that binds to transcriptional enhancers. Science 1989, 243:629-635.

27. Wedel A, Weiss DS, Popham D, Dröge PP, Kustu S: A bacterial enhancer functions to tether a transcriptional activator near a promoter. Science 1990, 248:486-490

28. Guarino LA, Summers MD: Interspersed homologous DNA of Autographa californica nuclear polyhedrosis virus enhances delayed-early gene expression. J Virol 1986, 60:215-223.

29. Olson VA, Wetter JA, Friesen PD: The highly conserved basic domain I of baculovirus IE1 is required for hr enhancer DNA binding and hrdependent transactivation. J Virol 2003, 77:5668-5677.

30. Farr A, Roman A: A pitfall of using a second plasmid to determine transfection efficiency. Nucleic Acids Res 1992, 20:920.

doi:10.1186/1743-422X-7-32

Cite this article as: Lin et al:: Baculovirus immediately early 1 , a mediator for homologous regions enhancer function in trans. Virology Journal 2010 7:32. 